RA. Telomere dysfunction and evolution of intestinal carcinoma in mice and humans. Nat Genet. 2001;28(2):155-159.

10. Roth A, Harley CB, Baerlocher GM. Imetelstat (GRN163L)-telomerase-based cancer therapy. Recent Results Cancer Res. 2010;184:221-234.

11. Begus-Nahrmann Y, et al. Transient telomere dysfunction induces chromosomal instability and promotes carcinogenesis. J Clin Invest. 2012; 122(6):2283-2288

12. Karlseder J, Broccoli D, Dai Y, Hardy S, de Lange T. p53- and ATM-dependent apoptosis induced by telomeres lacking TRF2. Science. 1999; 283(5406):1321-1325.

13. Plentz RR, et al. Telomere shortening correlates with increasing aneuploidy of chromosome 8 in human hepatocellular carcinoma. Hepatology. 2005; 42(3):522-526.

14. Ding Z, et al. Telomerase reactivation following telomere dysfunction yields murine prostate tumors with bone metastases. Cell. 2012;148(5):896-907.

15. $\mathrm{Hu}$ J, et al. Antitelomerase therapy provokes ALT and mitochondrial adaptive mechanisms in cancer. Cell. 2012;148(4):651-663.

16. Horikawa I, et al. Differential cis-regulation of human versus mouse TERT gene expression in vivo: identification of a human-specific repressive element. Proc Natl Acad Sci U S A. 2005; 102(51):18437-18442.

17. Khoo CM, Carrasco DR, Bosenberg MW, Paik JH,
Depinho RA. Ink4a/Arf tumor suppressor does not modulate the degenerative conditions or tumor spectrum of the telomerase-deficient mouse. Proc Natl Acad Sci U S A. 2007;104(10):3931-3936.

18. Smogorzewska A, de Lange T. Different telomere damage signaling pathways in human and mouse cells. EMBO J. 2002;21(16):4338-4348.

19. Herbig U, Jobling WA, Chen BP, Chen DJ, Sedivy JM. Telomere shortening triggers senescence of human cells through a pathway involving ATM, p53, and p21(CIP1), but not p16(INK4a). Mol Cell. 2004;14(4):501-513.

20. Takai H, Smogorzewska A, de Lange T. DNA damage foci at dysfunctional telomeres. Curr Biol. 2003;13(17):1549-1556.

\title{
Therapeutic potential of a peptide targeting BCL-2 cell guardians in cancer
}

\author{
Jerry M. Adams \\ Walter and Eliza Hall Institute of Medical Research, Molecular Genetics of Cancer Division, Parkville, Victoria, Australia. University of Melbourne, \\ Department of Medical Biology, Parkville, Victoria, Australia.
}

\begin{abstract}
A promising approach to cancer therapy is to elicit apoptosis with "BH3 mimetic" drugs, which target proteins of the BCL-2 family. As of yet, however, such drugs can target only certain BCL-2 family proteins. Hence, in this issue of the JCI, LaBelle et al. assess instead the therapeutic potential of a "stapled" $\mathrm{BH} 3$ peptide from the BIM protein, which inactivates all its prosurvival relatives. The peptide killed cultured hematologic tumor cells and abated growth of a leukemia xenograft, without perturbing the hematopoietic compartment. Hence, such peptides might eventually provide a new way to treat refractory leukemias.
\end{abstract}

It is increasingly accepted that most, if not all, conventional cytotoxic cancer therapies rely upon eliciting programmed cell death (apoptosis) in the tumor cells, a process regulated principally by the BCL- 2 protein family (1). Interactions among the members of this family serve as a switch determining whether the cell will live or die. In response to intracellular damage, the distant cousins of BCL-2, called BH3-only proteins because they bear only the third of the four $\underline{B} C L-2$ homology domains that characterize this family, are activated and convey the cell death warrant. They use their $\mathrm{BH} 3$ domain to engage and neutralize their prosurvival relatives, e.g., BCL-2, BCL- $\mathrm{x}_{\mathrm{L}}$, and MCL-1, keeping these cell guardians from restraining the family's critical effector proteins, BAX and BAK. Once the guardians have been overcome, certain potent $\mathrm{BH} 3$-only proteins (e.g., BIM) can also directly acti-

Conflict of interest: The author has declared that no conflict of interest exists.

Citation for this article: J Clin Invest. 2012; 122(6):1965-1967. doi:10.1172/JCI64120. vate $\mathrm{BAX}$ and $\mathrm{BAK}$, which then damage the mitochondria and unleash the proteolytic cascade that culminates in cell death (1).

\section{The prospects of $\mathrm{BH} 3$ mimetics}

Oncologists have long dreamed of drugs that would directly flip the apoptotic switch in cancer cells. That dream inspired the development of the first $\mathrm{BH} 3$ mimetic drugs (reviewed in refs. 1,2), the best studied of which are ABT-737 (3) and its orally bioavailable derivative ABT-263 (4). These drugs are specific for certain prosurvival BCL-2 family members; for example, they bind BCL-2 and BCL- $\mathrm{x}_{\mathrm{L}}$ with high affinity but not MCL-1 (Figure 1A). Consequently, as single agents they kill cells whose survival depends primarily on BCL-2 and/or BCL- $\mathrm{x}_{\mathrm{L}}$ but not those containing sufficient MCL-1 to restrain BAX, unless another agent inactivates or eliminates MCL-1 (5). The many promising preclinical findings with ABT-737 and ABT-263 have led to clinical trials of the latter. Notably, with chronic lymphocytic leukemia, which is sustained by high levels of BCL-2, ABT-263 has shown substantial efficacy as a single agent in one-third of patients, even in cases refractory to all conventional therapies and with poor prognostic markers (6).

In vitro and in vivo, ABT-263 and ABT737 can greatly augment the action of diverse conventional chemotherapeutics $(3,4)$, most likely because those agents diminish active MCL-1 levels, but which drug combinations will be tolerable in the clinic remains unclear. For example, the ability of ABT-737 and ABT-263 to engage BCL- $\mathrm{X}_{\mathrm{L}}$, which is the principal guardian of platelet survival (7), provokes a transient thrombocytopenia, and that has proven to be the dose-limiting toxicity for ABT-263 (6). Consequently, a novel BH3-mimetic highly specific for BCL-2 (ABT-199), which has recently entered clinical trial, should permit higher doses and have even greater promise for chronic lymphocytic leukemia and other diseases sustained by BCL-2 (1).

Eventually, BH3 mimetics that effectively target other family members (e.g., MCL-1) are likely to be developed, but the path to such agents is arduous, because as yet no defined organic skeleton adequately mimics the $\alpha$-helix assumed by the 16 - to 26-residue $\mathrm{BH} 3$ domain upon binding to the hydrophobic groove on the surface of its prosurvival relatives. Hence, the development of a new $\mathrm{BH} 3$ mimetic typically commences with screens of some $10^{6}$ chemical building blocks for binding to the BCL-2 family protein target; the resulting weak "hits" must be modified iteratively over 
A

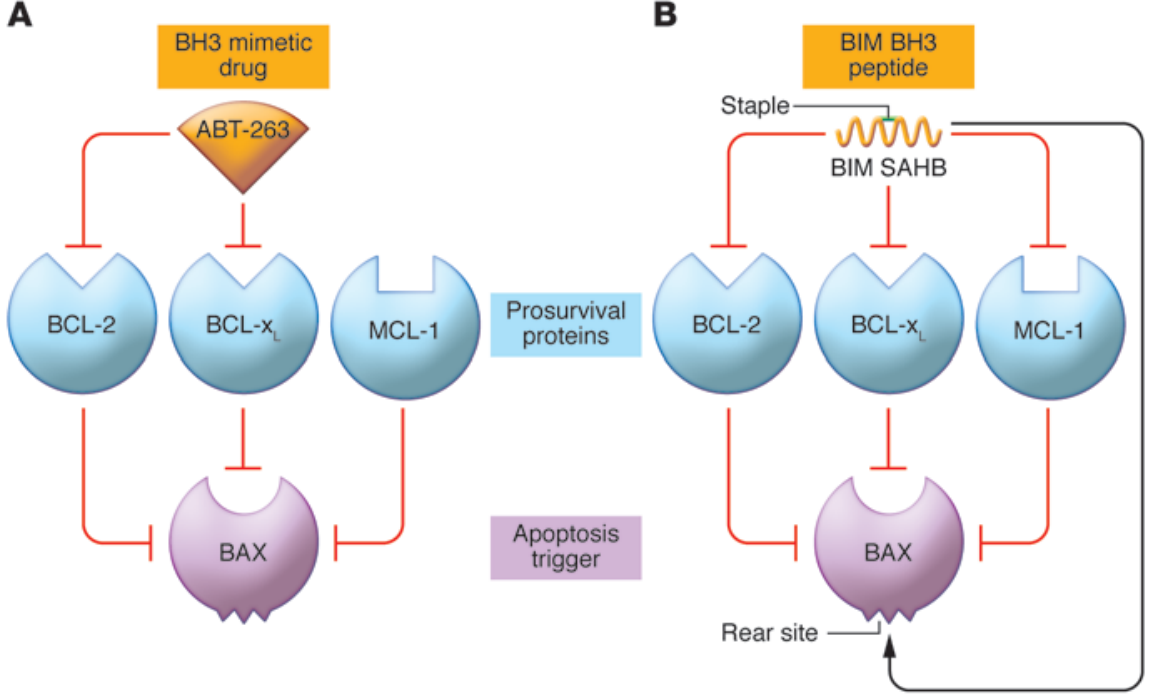

Figure 1

Contrasting pharmacologic strategies for reactivating cell death in refractory hematologic malignancies with a $\mathrm{BH} 3$ mimetic compound or a stabilized BIM BH3 peptide. (A) Because the $\mathrm{BH} 3$ mimetic ABT-263 effectively neutralizes BCL-2 and BCL- $x_{L}$ but not MCL-1, it is very effective as a single agent for malignancies in which $\mathrm{BCL}-2$ or $\mathrm{BCL}-\mathrm{X}_{\mathrm{L}}$ is the dominant guardian restraining BAX but not those in which MCL-1 can still restrain BAX and prevent apoptosis. (B) LaBelle et al. have investigated the apoptotic potential of a hydrocarbon stapled BH3 peptide (SAHB) from the protein BIM, which broadly targets BCL-2 family proteins and can directly activate $B A X$ as well as neutralize all its prosurvival relatives. In principle, the BIM SAHB could be more potent than a more specific $\mathrm{BH} 3$ mimetic, and, indeed, it proved to augment killing of hematologic tumor cells refractory to ABT-263, suggesting that such peptides might have potential as anticancer therapeutics.

many months or even years in myriad ways to obtain compounds with the high affinities (low nM range) of the $\mathrm{BH} 3$ peptides, and many such candidates prove to have unsuitable pharmacology or toxicology.

\section{BH3 peptides: potential and limitations}

Could this grueling route be truncated by using modified forms of the $\mathrm{BH} 3$ peptides themselves as anticancer drugs? That is the intriguing issue addressed by LaBelle et al. in this issue of the JCI (8). This group, directed by Loren Walensky, has previously developed forms of $\mathrm{BH} 3$ peptides in which a hydrocarbon staple bridges two nonessential residues separated by a helical turn to force the peptide into the $\alpha$-helical conformation that favors binding to BCL-2 family members (ref. 9 and Figure 1B). These so-called stabilized $\alpha$ helix of BCL-2 domains (SAHBs) are more stable than their unmodified counterparts and, unlike them, are readily taken up by cells (9). In previous studies, this group has shown that SAHBs derived from two of the most potent BH3-only proteins, BIM and BID, bind transiently to BAX and trigger the confor- mational changes that allow BAX to oligomerize and permeabilize the mitochondrial outer membrane $(10,11)$. Unexpectedly, the trigger site on BAX was mapped not to the hydrophobic groove resembling that in the prosurvival proteins but instead to a distal "rear site" (Figure 1B).

LaBelle et al. explore the therapeutic potential of a BIM SAHB (8). As expected from studies with unmodified BIM BH3 peptides $(12,13)$, the BIM SAHB, but not a point mutant control, bound in vitro with high affinity to all the prosurvival family members tested (Figure 1B). It was then tested on five cultured hematopoietic tumor cell lines that are relatively resistant to ABT-263. As well as entering the cells, the BIM SAHB, but not the mutant control, localized predominantly to the mitochondria, where many BCL-2 family members congregate. Notably, at low $\mu \mathrm{M}$ levels the BIM SAHB killed the cultured tumor cells but not nontransformed fibroblasts, and the cell death had all the hallmarks of apoptosis. However, the resistance of the fibroblasts calls into question the potency of the BIM SAHB, because BIM overexpression readily kills mouse fibroblasts (and many other cells), as does ABT-737 if MCL-1 is inactivated or deleted (5). Thus, it appears that the BIM SAHB does not encapsulate the full activity of the BIM polypeptide.

In the experiments most pertinent to clinical potential, mice bearing a human leukemia graft were treated with the BIM SAHB, both as a single agent and together with other cell death stimuli. On its own, the BIM SAHB retarded tumor growth significantly, albeit modestly $(50 \%)$. In combination with TRAIL, which elicits apoptosis through the extrinsic pathway, the peptide showed marked synergy. Its combination with ABT-263 also significantly slowed tumor growth, albeit not as dramatically as might have been expected, given that approximately $90 \%$ of cells were killed in vitro. As the mice received only a fixed SAHB dose, one wonders whether a several-fold higher level would have given a more marked result.

The study also assessed whether the BIM SAHB could reverse the lymphocytosis induced by knocking out the Bim gene (14) by treating mice whose hematopoietic compartment had been reconstituted with BIM-deficient cells. In the untreated mice, BIM-null B lymphocytes infiltrated the liver and kidney, perhaps because the absence of BIM allowed them to survive in these foreign environments without their normal cytokine support. The SAHB treatment killed many of these invading cells but not cells in the surrounding liver and kidney parenchyma. The treatment did not, however, ameliorate the increased spleen weight provoked by the absence of BIM. This suggests that, at least with the dosage used, the BIM SAHB incompletely restored BIM function.

\section{A window for peptide therapy?}

A particular concern about any therapy designed to stimulate cell death, whether through a $\mathrm{BH} 3$ mimetic or a SAHB, is that some normal tissues may be damaged unacceptably. It is therefore interesting to note that the SAHB treatments, alone or together with ABT-263, did not lower white blood cell numbers or body weight. This suggests that, at least for the moderate doses used, the treatment might be safe. Unfortunately, platelet numbers were not examined, as inhibition of BCL- $\mathrm{x}_{\mathrm{L}}$ by the SAHB would be expected to induce transient thrombocytopenia (7).

In conclusion, the work of LaBelle et al. suggests that stapled $\mathrm{BH} 3$ peptides could 
become a new weapon for the oncologist's armamentarium. An agent like BIM SAHB that targets the full panoply of prosurvival guardians, and is expected to also promote BAX activation directly, might have been expected to decimate many normal tissues. That was not observed under conditions in which a substantial proportion of tumor cells were killed. Presumably, the malignant cells are more sensitive than many normal cells, because the stresses induced by their disturbed cell cycle checkpoints have rendered them primed for apoptosis (13), perhaps by increasing their level of BIM. Thus, the results of the work by LaBelle et al. suggest that there may well be a therapeutic window for peptides like the BIM SAHB in treatment of refractory leukemias.

\section{Acknowledgments}

This work was supported by the National Health and Medical Research Council (NHMRC), Australia (program grant 461221 and fellowship 516703), and was facilitated by Victorian State Government Operational Infrastructure Support and Australian Government NHMRC IRIISS.

Address for correspondence: Jerry M. Adams, Walter and Eliza Hall Institute of Medical Research, 1G Royal Parade, Parkville 3052, Victoria, Australia. Phone: 61.3. 9345.2491; Fax: 61.3.9347.0852; E-mail: adams@wehi.edu.au.

1. Strasser A, Cory S, Adams JM. Deciphering the rules of programmed cell death to improve therapy of cancer and other diseases. EMBOJ. 2011;30(18):3667-3683.

2. Lessene G, Czabotar PE, Colman PM. BCL-2 family antagonists for cancer therapy. Nat Rev Drug Discov. 2008;7(12):989-1000

3. Oltersdorf T, et al. An inhibitor of Bcl-2 family proteins induces regression of solid tumours. Nature. 2005;435(7042):677-681

4. Tse C, et al. ABT-263: a potent and orally bioavailable Bcl-2 family inhibitor. Cancer Res. 2008; 68(9):3421-3428.

5. van Delft MF, et al. The BH3 mimetic ABT-737 targets selective $\mathrm{Bcl}-2$ proteins and efficiently induces apoptosis via Bak/Bax if Mcl-1 is neutralized. Cancer Cell. 2006;10(5):389-399.

6. Roberts AW, et al. Substantial susceptibility of chronic lymphocytic leukemia to BCL2 inhibition: results of a phase I study of navitoclax in patients with relapsed or refractory disease.J Clin Oncol. 2012; 30(5):488-496.

7. Mason KD, et al. Programmed anuclear cell death delimits platelet life span. Cell. 2007; 128(6):1173-1186

8. LaBelle JL, et al. A stapled BIM peptide overcomes apoptotic resistance in hematologic cancers. J Clin Invest. 2012;122(6):2018-2031.

9. Walensky LD, et al. Activation of apoptosis in vivo by a hydrocarbon-stapled BH3 helix. Science. 2004;305(5689):1466-1470.

10. Gavathiotis E, et al. BAX activation is initiated at a novel interaction site. Nature. 2008; 455(7216):1076-1081.

11. Gavathiotis E, Reyna DE, Davis ML, Bird GH, Walensky LD. BH3-triggered structural reorganization drives the activation of proapoptotic BAX. Mol Cell. 2010;40(3):481-492.

12. Chen L, et al. Differential targeting of pro-survival $\mathrm{Bcl}-2$ proteins by their $\mathrm{BH} 3$-only ligands allows complementary apoptotic function. Mol Cell. 2005;17(3):393-403.

13. Certo $M$, et al. Mitochondria primed by death signals determine cellular addiction to antiapoptotic BCL-2 family members. Cancer Cell. 2006;9(5):351-365.

14. Bouillet $\mathrm{P}$, et al. Proapoptotic $\mathrm{Bcl}-2$ relative Bim required for certain apoptotic responses, leukocyte homeostasis, and to preclude autoimmunity. Science. 1999;286(5445):1735-1738.

\title{
Taming endothelial activation with a microRNA
}

\author{
Jason E. Fish and Myron I. Cybulsky
}

\begin{abstract}
Division of Cellular and Molecular Biology, Toronto General Research Institute, University Health Network, Toronto, Ontario, Canada. Department of Laboratory Medicine and Pathobiology, University of Toronto, Toronto, Ontario, Canada. Heart and Stroke Richard Lewar Centre of Excellence in Cardiovascular Research, Toronto, Ontario, Canada.
\end{abstract}

\begin{abstract}
Inflammation plays an essential role in vascular pathologies, including those associated with sepsis and atherosclerosis. Identifying negative regulators of inflammatory signaling pathways may provide novel therapeutic targets for these diseases. In this issue of the JCI, Sun et al. show that in endothelial cells, microRNA-181b (miR-181b) plays a vital role in controlling inflammation by targeting importin- $\alpha 3$, a regulator of NF- $\kappa B$ nuclear import. These findings provide compelling evidence that modulation of microRNAs may be a useful therapeutic approach for inflammatory vascular diseases.
\end{abstract}

\section{Vascular endothelium and inflammation}

The vascular endothelium forms the interface between blood and tissues and plays a critical and active role in maintaining blood vessel and tissue homeostasis as well as a nonthrombotic and nonadhesive vascular surface. Endothelial cell activation by proinflammatory stimuli promotes leukocyte recruitment from the blood into extravascular tissues and thus contributes to the

Conflict of interest: The authors have declared that no conflict of interest exists.

Citation for this article: J Clin Invest. 2012; 122(6):1967-1970. doi:10.1172/JCI63818. pathogenesis of various inflammatory diseases (1). Multiple signaling pathways participate in this process, but the NF-кB signaling pathway plays a particularly central role. NF- $\mathrm{KB}$ signal transduction in endothelium culminates in the expression of multiple proinflammatory genes, including cell adhesion molecules such as E- and P-selectin, vascular cell adhesion molecule 1 (VCAM-1), and intercellular adhesion molecule 1 (ICAM-1), as well as various chemokines and cytokines. Since endothelial cell activation contributes to the pathogenesis of multiple inflammatory and immune conditions, targeting NF- $\mathrm{KB}$ signal transduction is an attractive poten- tial therapeutic strategy. However, enthusiasm should be tempered because a subset of NF-кB target genes promotes cell survival under conditions of stress. It is also important to target the "right" cells because inhibition of NF- $\mathrm{KB}$ signaling in endothelial cells reduces atherosclerosis, whereas inhibition in macrophages promotes cell death and exacerbates atherosclerosis $(2,3)$. In this issue of the JCI, Sun et al. (4) explore a novel microRNA-based approach to regulating NF- $\mathrm{KB}$ signaling and provide insights into its therapeutic utility.

\section{NF-KB signal transduction}

The NF- $\mathrm{B} /$ Rel family consists of protein homo- and heterodimers, each with different DNA binding and activation specificity (5). The $\mathrm{p} 50 / \mathrm{p} 65$ heterodimer is the prototypic NF- $\kappa \mathrm{B}$ and is predominant in endothelial cells. In quiescent cells, NF-кB is predominantly localized in the cytoplasm, where it is retained through association with an inhibitor protein (IкB) (ref. 5 and Figure 1). There are several IкBs, and 